\title{
Coalitional power indices applied to voting systems
}

\author{
Xavier Molinero $^{1}$, Joan Blasco ${ }^{2}$ \\ ${ }^{1}$ Mathematics Department, Universitat Politècnica de Catalunya·BarcelonTech \\ Barcelona Graduate School of Mathematics \\ ${ }^{2}$ Barcelona School of Informatics, Universitat Politècnica de Catalunya·BarcelonTech \\ xavier.molinero@upc.edu, joan.blasco@est.fib.upc.edu
}

\begin{abstract}
Keywords: Simple games, Coalitional power indices, Voting systems
Abstract: We describe voting mechanisms to study voting systems. The classical power indices applied to simple games just consider parties, players or voters. Here, we also consider games with a priori unions, i.e., coalitions among parties, players or voters. We measure the power of each party, player or voter when there are coalitions among them. In particular, we study real situations of voting systems using extended Shapley-Shubik and Banzhaf indices, the so-called coalitional power indices. We also introduce a dynamic programming to compute them.
\end{abstract}

\section{INTRODUCTION}

Classical cooperative games provide mathematical tools useful to study situations of conflict of interest and cooperation arising from the real world. A field where cooperative games have been frequently applied is political science. By using simple games, collective decision-making mechanisms ruled by voting have often been described and analyzed (Carreras, 2004). The value theory (one of the main streams of the cooperative game theory) has given rise, by restricting it to simple games, to a "power index theory". The most conspicuous representatives of this line of research are the Shapley-Shubik (Shapley and Shubik, 1954) and the Banzhaf-Coleman (Penrose, 1946; Banzhaf III, 1964; Coleman, 1971; Owen, 1975; Moshe'Machover Dan, 1998) power indices. A survey about power indices can be found in (Freixas, 2010).

However, it seems that this framework does not suffice to analyze all aspects of voting. Following early initial papers by Lucas and Thrall (Thrall and Lucas, 1963) and Myerson (Myerson, 1977) who, respectively, generalized cooperative games in characteristic function form to "games in partition function form" and extended the Shapley value (Shapley, 1953) to this new class of games, Bolger (Bolger, 1983) used a Banzhaf-type power index for the so-called "multicandidate voting games". Bolger (Bolger, 1993) also defined and axiomatically characterized a new extension of the Shapley value rela- tively to each alternative. We agree that this relativity is an essential feature of any evaluation of games with alternatives.

In classical simple games it is implicitly assumed that players can join them to form coalitions or strategies (Holler and Owen, 2013; Carreras and Owen, 1988; Owen, 1977a; Fagen, 1963). The goal of this paper is to show the wide possibilities of games with coalitions among players to represent real voting systems. We consider real situations and we study the exact role of each player. The analysis is done with the classical Shapley-Shubik and Banzhaf power indices with coalitions. Note that some definitions and concepts have been gotten from previous works as (Carreras and Magaña, 2008; Nieto, 1996), among others.

\section{CLASSICAL COALITIONAL POWER INDICES FOR SIMPLE GAMES}

This section considers Shapley-Shubik and Banzhaf power indices, as well as coalitional Shapley-Shubik and Banzhaf power indices. Next we introduce some references and definitions related with them.

Simple games can be viewed as models of voting systems in which a single alternative, such as a bill or an amendment, is pitted against the status quo.

Definition 1 ((Freixas and Molinero, 2009)). A simple game is a pair $(N, v)$ in which $N=\{1,2, \ldots, n\}$ and 
$v(S)=1$ or 0 , where $S$ in a subset of $N$, that satisfies: (1) $v(N)=1$, (2) $v(\emptyset)=0$ and (3) the monotonicity property: $v(s)=1$ and $S \subseteq T \subseteq N$ implies $v(T)=1$.

So far, a coalition has represented a set of agents that worked on its own. In a coalition structure (CS), the different coalitions are intended to work independently of each other. We can also interpret a coalition to represent a group of agent that is more likely to work together within a larger group of agents (because of personal or political affinities). The members of a coalition do not mind working with other agents, but they want to be together and negotiate their payoff together, which may improve their bargaining power. This is the idea used in games with a priori unions. Formally, a game with a priori unions is similar to a game with CS: it consists of a triplet $(N, v, \mathcal{B})$ where $(N, v)$ is a simple game ${ }^{1}$ and $\mathcal{B}=\left\{B_{1}, B_{2}, \ldots, B_{m}\right\}$ is a CS defined over the set of players, $N$.

Based on Shapley's value, Owen characterizes axiomatically a new value, now known as Owen's coalitional value or simply coalitional value, which reflects the different possibilities of each player depending on the subset of the coalition structure to which he belongs. In the development of this idea, the possibility that the different blocks of the coalition structure cooperate with each other is not excluded. On the contrary, it is considered that each block chooses a representative, $k$ for the $B_{k}$ block, and these are the ones that negotiate (play). If $M=\{1,2, \ldots, m\}$ is the set formed by the representatives of the blocks of $\mathcal{B}$, then the coalitional value of a player $i \in B_{k}$ is:

Definition 2 ((Owen, 1977b)). The Owen's coalitional value is

$$
\begin{aligned}
\phi_{i}[v ; \mathcal{B}]=\sum_{S \subseteq B_{k}} \sum_{T \subseteq M} & \frac{(t-1) !(m-t) !}{m !} . \\
& \frac{(s-1) !\left(b_{k}-s\right) !}{b_{k} !} . \\
& {\left[v^{S}(T)-v^{S}(T \backslash\{i\})\right] }
\end{aligned}
$$

where $t=|T|, m=|M|, s=|S|$ and $b_{k}=\left|B_{k}\right|$.

Later, Owen also defined the corresponding coalitional value for Banzhaf power index as follows.

Definition 3 ((Owen, 1981)). The Banzhaf-Owen's coalitional value is

$$
\begin{aligned}
\psi_{i}[v ; \mathcal{B}]=\sum_{S \subseteq B_{k}} \sum_{T \subseteq M} & \frac{1}{2^{m-1}} \cdot \frac{1}{2^{b_{k}-1}} . \\
& {\left[v^{S}(T)-v^{S}(T \backslash\{i\})\right] }
\end{aligned}
$$

where $t=|T|, m=|M|, s=|S|$ and $b_{k}=\left|B_{k}\right|$.

\footnotetext{
${ }^{1}$ Note that it also works for cooperative games (see (Taylor and Zwicker, 1999)).
}

Note that Owen's coalitional and Banzhaf-Owen's coalitional power indices are also called coalitional Shapley-Shubik and coalitional Banzhaf power indices, respectively.

\section{THE IMPLEMENTATION OF CLASSICAL COALITIONAL POWER INDICES}

Using all the information from the previous section, Formulas 1 and 2, we have done a program that computes coalitional Shapley-Shubik and coalitional Banzhaf power indexes.

The basic case for this formula is when every party is in a coalition by itself, i.e., $B_{k}=\{k\}$ for $1 \leq k \leq n=m$. Then, the formula is the same as the one for simple games without coalitions. The algorithm uses backtracking to get the $2^{b_{k}+m-2}$ different combinations. The function will have $b_{k}+m-2$ levels but this time the options are between getting that player or ignoring it.

We divided our function in two parts.

The first one chooses all combinations with $b_{k}$ players inside the coalition $B_{k}$ in which our player $i$ belongs to, i.e., $i \in B_{k}$. See Figure 1 .

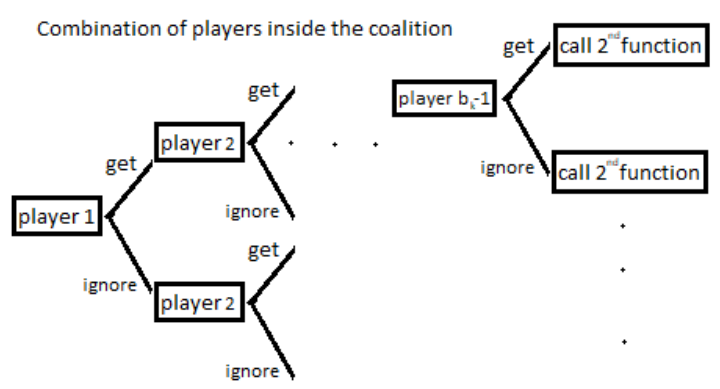

Figure 1: Combinations of players inside of $B_{k}$.

Afterwards, we call the $2^{\text {nd }}$ function. This function considers all combinations with $m$ indices inside $M$ including the index $j \in M$ which our player $i$ belongs to, i.e., $i \in B_{j}$. See Figure 2.

\subsection{Dynamic programming}

In this section we just consider the so-called weighted majority games, a subclass of simple games defined as follows.

Definition 4 ((Freixas and Molinero, 2009)). A weighted majority game is a simple game $(N, v)$, where there exists a representation 


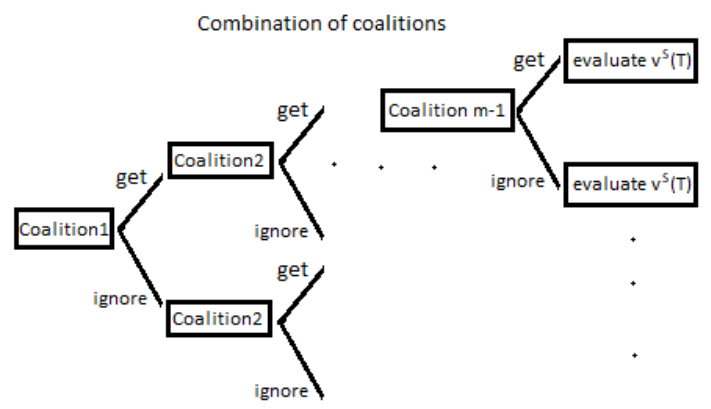

Figure 2: Combinations of indices inside of $M$.

$\left(q, w=\left\{w_{1}, \ldots, w_{n}\right\}\right)$ such that $v(S)=1$ if and only if $w(S) \geq q$, where $w(S)=\sum_{i \in S} w_{i}$.

There is an algorithm that uses dynamic programming to calculate the Shapley-Shubik and Banzhaf indices, for weighted majority games, with a time complexity of $O\left(n^{2} q\right)$ and space requirement of $O(n q)$ for a defined quota $q$ (Lucas, 1983; Brams and Affuso, 1976).

We extended the previous dynamic algorithm introduced for simple games (Lucas, 1983; Brams and Affuso, 1976) to one that can use coalitions.

Our first step in our algorithm is to reshape our $n$ players divided in $m$ coalitions into $n^{\prime}$ players that will serve as our input.

Those $n^{\prime}$ players will be composed for the $b_{k}-1$ players in our coalition and the $m-1$ coalitions that we will transform into players $c_{i}$ with the weight of the whole coalition.

$$
N^{\prime}=\left\{p_{1}, \ldots, p_{b_{k}-1}\right\} \cup\left\{c_{1}, \ldots, c_{m-1}\right\}
$$

In which:

$$
p(S)=|V|, V \subseteq S, \forall x \in V, x \in B_{k}
$$

Then we will make a partition of $N^{\prime}$ players into subsets $N_{1}^{\prime}, N_{2}^{\prime}, \ldots, N_{z}^{\prime}$ satisfying that:

1. $N_{1}^{\prime} \cup N_{2}^{\prime} \cup \ldots \cup N_{z}^{\prime}=N^{\prime}, N_{x}^{\prime} \cap N_{y}^{\prime}=\emptyset(x \neq y)$,

2. For all $x, y: 1 \leq x<y \leq z, \forall i \in N_{x}^{\prime}, \forall j \in N_{y}^{\prime}, w_{i}>$ $w_{j}$

3. For all $x: 1 \leq x \leq z, \forall i, j \in N_{x}^{\prime}, w_{i}=w_{j}$.

For every player $i, \hat{c}_{i}(w, t, s, x)$ denotes the number

$$
\begin{aligned}
& \#\{S \subseteq N^{\prime}-\{i\}: w(S)=w,|S|=t, p(S)=s, \\
&\left.S \cap N_{x}^{\prime} \neq \emptyset, S \cap N_{x+1}^{\prime}=\ldots=S \cap N_{z}^{\prime}=\emptyset\right\} .
\end{aligned}
$$

and, for every player $i, c_{i}(w, t, x)$ denotes the number

$$
\begin{array}{r}
\#\left\{S \subseteq N^{\prime}-\{i\}: w(S)=w,|S|=t, S \cap N_{x}^{\prime} \neq \emptyset,\right. \\
\left.S \cap N_{x+1}^{\prime}=\ldots=S \cap N_{z}^{\prime}=\emptyset\right\} .
\end{array}
$$

Then the Owen $(\phi)$ and Banzhaf-Owen $(\psi)$ coalitional power indices of the player $i \in N_{y}$ are described as follows,

$$
\begin{array}{r}
\phi_{i}[v ; \mathcal{B}]=\sum_{t=1}^{n^{\prime}-1} \sum_{w=q-w_{i}}^{q-1} \sum_{x=1}^{z} \sum_{s=1}^{b_{k}} \frac{(t-s) !(m-(t-s)-1) !}{m !} . \\
\frac{s !\left(b_{k}-s-1\right)}{b_{k} !} . \\
\hat{c}_{i}(w, t, s, x),
\end{array}
$$

and

$$
\psi_{i}[v ; \mathcal{B}]=\frac{1}{2^{m-1}} \cdot \frac{1}{2^{b_{k}-1}} \cdot \sum_{t=1}^{n-1} \sum_{w=q-w_{i}}^{q-1} \sum_{x=1}^{z} c_{i}(w, t, x),
$$

respectively.

Note that $v(j)$ is the number of combinations getting $j$ elements from the $s^{\prime}$ elements, times, the combinations getting $y-j$ elements from $y^{\prime}-s^{\prime}$.

Then we can get an algorithm with time complexity of $O\left(\left(n^{\prime} b_{k}\right)^{2} q\right)$ and space requirement of $O\left(n^{\prime} q\right)$.

This algorithm improves the time to calculate our index when the number of players grows but it becomes slower when the quota is too big. To reduce the quota we can try to apply Greatest Common Divisor to the weight of our players and the quota.

Nevertheless the biggest parliament in the world is China's National People's Congress with 2890 seats in which the biggest party controls the $71.1 \%$ of the seats. This parliament is followed by the UK Upper House with 793 and the European Parliament with 751 seats. So, in the worst case scenario where China needs a quota of $75 \%$ and there will be $n$ players in $B_{k}$ our algorithm will have a time cost of $O\left(1987 n^{3}\right)$. That means it will start to be faster than the exponential version for $n>24$. See Figure 3 .

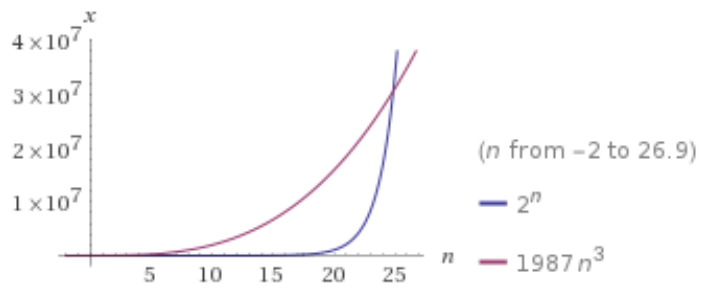

Figure 3: Complexity of the standard $\left(O\left(2^{n}\right)\right)$ and the dynamic programming $\left(O\left(1987 n^{3}\right)\right)$ algorithms.

For the parliament of the UK Upper House, the average parliament has 300 seats, with a quota of $75 \%$ and half of the players in the coalition, our algorithm will have a time cost of $O\left(100 n^{3}\right)$. So our algorithm will act faster than the exponential version for $n>19$ .See Figure 4. 


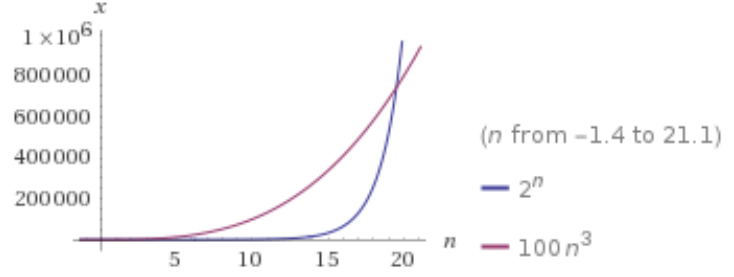

Figure 4: Complexity of the standard $\left(O\left(2^{n}\right)\right)$ and the dynamic programming $\left(O\left(100 n^{3}\right)\right)$ algorithms.

\section{REAL VOTING SYSTEMS}

To success a game (the approval or defeat of a motion), a number of players need to agree in the same decision to reach a certain quorum amount of votes. Here we mention three different scenarios.

- Voting a motion that requires a qualified majority of 2/3 of the players. This kind of motion is necessary to reform the constitution in the Spanish parliament.

- Voting a motion that requires an absolute majority of $1 / 2$ of the players. This motion is used for a censure motion in the Spanish parliament.

- Voting a motion that requires a relative majority, i..e, more votes for the motion than against it. This is the most common form of voting and the one in which abstention plays a different role than in the other two cases because we do not take into account abstention votes, i.e., we just compare the votes in favour with the votes against.

We study now two different voting rules: Catalan Parliament 2019 and German Parliament 2019,

\subsubsection{Catalan Parliament 2019}

$\phi_{i}\left[v^{q}, \mathcal{B}\right]$ is the coalitional Owen's power index for the player $i$, with quota $q$ and structure of coalitions

$$
\begin{array}{r}
\mathcal{B}=\left\{\{J U N T S x C A T, E R C\},\left\{C^{\prime} s\right\},\{P S C\},\right. \\
\{E n C o m u\},\{C U P\},\{P P\}\},
\end{array}
$$

see Table 1, and

$$
\begin{array}{r}
\mathcal{B}=\left\{\{J U N T S x C A T, E R C, C U P\},\left\{C^{\prime} s\right\},\{P S C\},\right. \\
\{E n C o m u\},\{P P\}\},
\end{array}
$$

see Table 2.

In the government in catalonia 2019 it was a coalition between JUNTSxCAT and ERC (Table 1), but if CUP, which had similar political interest, was added to the coalition they will have a complete majority.

\begin{tabular}{ccccc} 
party & weight & $\%$ weight & $\phi_{i}\left[v^{2 / 3}, \mathcal{B}\right]$ & $\phi_{i}\left[v^{1 / 2}, \mathcal{B}\right]$ \\
\hline C's & 36 & $26.66 \%$ & 21.6667 & 6.6667 \\
JUNTSxCAT & 34 & $25.19 \%$ & 31.6667 & 33.3333 \\
ERC & 32 & $23.70 \%$ & 38.3333 & 33.3333 \\
PSC & 17 & $12.59 \%$ & 10 & 6.6667 \\
EnComu & 8 & $5.92 \%$ & 5 & 6.6667 \\
CUP & 4 & $2.96 \%$ & 1.6667 & 6.6667 \\
PP & 4 & $2.96 \%$ & 1.6667 & 6.6667 \\
\hline
\end{tabular}

Table 1: Power distribution in the Catalan Parliament 2019 using coalitions $B_{1}=\{J U N T S x C A T, E R C\}$.

\begin{tabular}{ccccc} 
party & weight & $\%$ weight & $\phi_{i}\left[v^{2 / 3}, \mathcal{B}\right]$ & $\phi_{i}\left[v^{1 / 2}, \mathcal{B}\right]$ \\
\hline C's & 36 & $26.66 \%$ & 20 & 0 \\
JUNTSxCAT & 34 & $25.19 \%$ & 31.6667 & 43.3333 \\
ERC & 32 & $23.70 \%$ & 38.3333 & 43.3333 \\
PSC & 17 & $12.59 \%$ & 11.6667 & 0 \\
EnComu & 8 & $5.92 \%$ & 3.3333 & 0 \\
CUP & 4 & $2.96 \%$ & 2.5 & 13.3333 \\
PP & 4 & $2.96 \%$ & 3.3333 & 0 \\
\hline
\end{tabular}

Table 2: Power distribution in the Catalan Parliament 2019 using coalitions $B_{1}=\{J U N T S x C A T, E R C, C U P\}$.

\subsubsection{German Parliament 2019}

$\psi_{i}\left[v^{q}, \mathcal{B}\right]$ denotes the coalitional Banzhaf-Owen's power index for the player $i$, with quota $q$ and structure of coalitions $\mathcal{B}$. Tables 3 and 4 analyze the German parliament 2019 using the Banzhaf-Owen coalitional value for $\mathcal{B}$ without coalitions and

$$
\begin{array}{r}
\mathcal{B}=\{\{C D U, S P D, C S U\},\{A f D\},\{F D P\}, \\
\{D I E L I N K E\},\{G R \ddot{U} N E\}\},
\end{array}
$$

which is the current coalition in the government, respectively.

\begin{tabular}{ccccc} 
party & weight & $\%$ weight & $\psi_{i}\left[v^{2 / 3}, \mathcal{B}\right]$ & $\psi_{i}\left[v^{1 / 2}, \mathcal{B}\right]$ \\
\hline CDU & 200 & $28.21 \%$ & 30.4348 & 29.0323 \\
SPD & 153 & $21.58 \%$ & 21.7391 & 19.3548 \\
AfD & 94 & $13.26 \%$ & 13.0435 & 16.129 \\
FDP & 80 & $11.28 \%$ & 10.7696 & 9.6774 \\
DIE LINKE & 69 & $9.73 \%$ & 8.6956 & 9.6774 \\
GRÜNE & 67 & $9.45 \%$ & 8.6956 & 9.6774 \\
CSU & 46 & $6.49 \%$ & 6.5217 & 6.4516 \\
\hline
\end{tabular}

Table 3: Power distribution in the German Parliament (Bundestag) 2019 without coalitions.

\begin{tabular}{ccccc} 
party & weight & $\%$ weight & $\psi_{i}\left[v^{2 / 3}, \mathcal{B}\right]$ & $\psi_{i}\left[v^{1 / 2}, \mathcal{B}\right]$ \\
\hline CDU & 200 & $28.21 \%$ & 32.5581 & 52.9412 \\
SPD & 153 & $21.58 \%$ & 23.2558 & 35.2941 \\
AfD & 94 & $13.26 \%$ & 13.9535 & 0 \\
FDP & 80 & $11.28 \%$ & 13.9535 & 0 \\
DIE LINKE & 69 & $9.73 \%$ & 4.6512 & 0 \\
GRÜNE & 67 & $9.45 \%$ & 4.6512 & 0 \\
CSU & 46 & $6.49 \%$ & 6.9767 & 11.7647 \\
\hline
\end{tabular}

Table 4: Power distribution in the German Parliament (Bundestag) 2019 using coalitions $B_{1}=\{C D U, S P D, C S U\}$.

Note that if we take into account the coalition that forms the government, the power of each party 
changes a lot, specially if that coalition has absolute majority.

\section{CONCLUSIONS AND FUTURE WORK}

We have computed some specific measures to establish the power that each party in a parliament holds, taking special attention when coalitions appears. In this vein, we have implemented the extension of Shapley-Shubik and Banzhaf power indices with coalitions. We have also studied more real voting systems in deep.

For our future work we are also planning on extending the considered definitions and algorithms. It could express multiple alternatives (as abstention) between input options, voting likeness (two parties with opposed ideologies are less likely to vote the same), and try to put both concepts together.

We also plan to improve the complexity of our algorithm (by the way, all of them are NP-Hard) using approximation methods for computing power indices (Fatima et al., 2012; Bachrach et al., 2010) and another methods (Alonso-Meijide and Bowles, 2005).

\section{ACKNOWLEDGEMENTS}

X. Molinero has been partially supported by funds from the Spanish Ministry of Economy and Competitiveness (MINECO) and the European Union (FEDER funds) under grants MTM2015-66818-P (VOTA-COOP) and MDM-2014-044 (BGSMath).

\section{REFERENCES}

Alonso-Meijide, J. M. and Bowles, C. (2005). Generating functions for coalitional power indices: An application to the imf. Annals of Operations Research, 137(1):21-44.

Bachrach, Y., Markakis, E., Resnick, E., Procaccia, A. D., Rosenschein, J. S., and Saberi, A. (2010). Approximating power indices: theoretical and empirical analysis. Autonomous Agents and Multi-Agent Systems, 20(2):105-122.

Banzhaf III, J. F. (1964). Weighted voting doesn't work: A mathematical analysis. Rutgers L. Rev., 19:317.

Bolger, E. M. (1983). The banzhaf index for multicandidate presidential elections. SIAM Journal on Algebraic Discrete Methods, 4(4):442-458.

Bolger, E. M. (1993). A value for games withn players andr alternatives. International Journal of Game Theory, 22(4):319-334.
Brams, S. J. and Affuso, P. J. (1976). Power and size: A new paradox. Theory and Decision, 7(1-2):29-56.

Carreras, F. (2004). $\alpha$-Decisiveness in Simple Games, pages 77-91. Springer US, Boston, MA.

Carreras, F. and Magaña, A. (2008). The shapley-shubik index for simple games with multiple alternatives. Annals of Operations Research, 158(1):81-97.

Carreras, F. and Owen, G. (1988). Evaluation of the catalonian parliament, 1980-1984. Mathematical Social Sciences, 15(1):87-92.

Coleman, J. S. (1971). Control of collectivities and the power of a collectivity to act. Social choice, pages 269-300.

Fagen, R. R. (1963). The theory of political coalitions. American Political Science Review, 57(2):446-447.

Fatima, S., Wooldridge, M., and Jennings, N. R. (2012). A heuristic approximation method for the banzhaf index for voting games. Multiagent and Grid Systems, 8(3):257-274.

Freixas, J. (2010). Power indices. Wiley Encyclopedia of Operations Research and Management Science.

Freixas, J. and Molinero, X. (2009). Simple games and weighted games: A theoretical and computational viewpoint. Discrete Appl. Math., 157(7):1496-1508.

Holler, M. J. and Owen, G. (2013). Power Indices and Coalition Formation. Springer US, Boston, MA.

Lucas, W. F. (1983). Measuring power in weighted voting systems. In Political and related models, pages 183238. Springer.

Moshe'Machover Dan, S. F. (1998). The measurement of voting power: theory and practice, problems and paradoxes.

Myerson, R. B. (1977). Values of games in partition function form. International Journal of Game Theory, 6(1):23-31.

Nieto, A. M. (1996). Formación de coaliciones en los juegos cooperativos y juegos con múltiples alternativas. $\mathrm{PhD}$ thesis, Universitat Politècnica de Catalunya.

Owen, G. (1975). Multilinear extensions and the banzhaf value. Naval research logistics quarterly, 22(4):741750 .

Owen, G. (1977a). Values of games with a priori unions. In Henn, R. and Moeschlin, O., editors, Mathematical Economics and Game Theory, pages 76-88, Berlin, Heidelberg. Springer Berlin Heidelberg.

Owen, G. (1977b). Values of games with a priori unions. In Mathematical economics and game theory, pages 76-88. Springer.

Owen, G. (1981). Modification of the banzhaf-coleman index for games with a priori unions. In Power, voting, and voting power, pages 232-238. Springer.

Penrose, L. S. (1946). The elementary statistics of majority voting. Journal of the Royal Statistical Society, 109(1):53-57.

Shapley, L. S. (1953). A value for n-person games. Contributions to the Theory of Games, 2(28):307-317.

Shapley, L. S. and Shubik, M. (1954). A method for evaluating the distribution of power in a committee system. American political science review, 48(3):787-792. 
Taylor, A. and Zwicker, W. (1999). Simple games: Desirability relations, trading, pseudoweightings. Princeton University Press, Princeton, NJ.

Thrall, R. M. and Lucas, W. F. (1963). N-person games in partition function form. Naval Research Logistics Quarterly, 10(1):281-298. 